\title{
Can Soft Models Describe Polymer Knots?
}

\author{
Jianrui Zhang, Hendrik Meyer, Peter Virnau, and Kostas Ch. Daoulas*
}

Cite This: Macromolecules 2020, 53, 10475-10486

Read Online

ABSTRACT: Similar to macroscopic ropes and cables, long polymers create knots. We address the fundamental question whether and under which conditions it is possible to describe these intriguing objects with crude models that capture only mesoscale polymer properties. We focus on melts of long polymers which we describe by a model typical for mesoscopic simulations. A wormlike chain model defines the polymer architecture. To describe nonbonded interactions, we deliberately choose a generic "soft" repulsive potential that leads to strongly overlapping monomers and coarse local liquid structure. The soft model is parametrized to

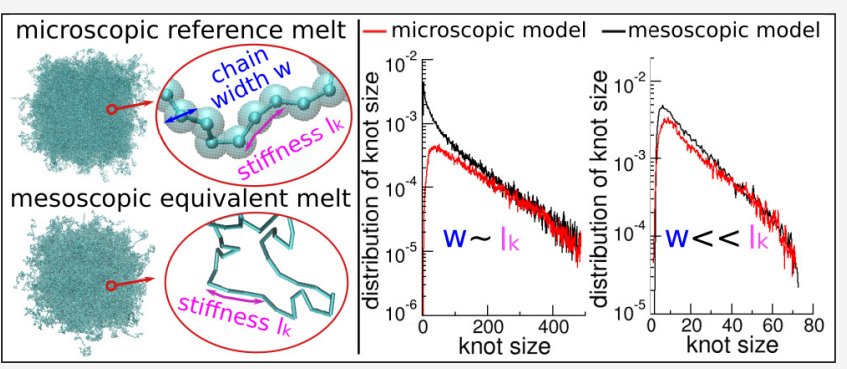
accurately reproduce mesoscopic structure and conformations of reference polymer melts described by a microscopic model. The microscopically resolved samples retain all generic features affecting polymer topology and provide, therefore, reliable reference data on knots. We compare characteristic knotting properties in mesoscopic and microscopically resolved melts for different cases of chain stiffness. We conclude that mesoscopic models can reliably describe knots in those melts, where the length scale characterizing polymer stiffness is substantially larger than the size of monomer-monomer excluded volume. In this case, simplified local liquid structure influences knotting properties only marginally. In contrast, mesoscopic models perform poorly in melts with flexible chains. We qualitatively explain our findings through a free energy model of simple knots available in the literature.

\section{INTRODUCTION}

Mesoscopic models of polymers are constructed by substituting a large number of microscopic degrees of freedom by a single effective interaction center. Such models are indispensable for studying polymeric materials on scales between a few and up to several hundreds of nanometers, benefiting from efficient sampling of configurational space. This efficiency stems from reduced amount of degrees of freedom and effective potentials that are comparable in strength to the thermal energy. This "softening" of interactions mitigates computational bottlenecks caused by microscopic hard excluded volume and is a consequence ${ }^{1-3}$ of the formal statistical mechanics of coarse-graining.

The contribution of mesoscopic models to basic theoretical understanding of polymeric materials has been significant, and representative examples are available in various topical reviews. $^{3-11}$ Moreover, mesoscopic simulations are a key element of algorithms developed for hierarchical modeling of polymeric materials, where equilibrated samples described by soft models are used to recover the microscopic description through efficient fine-graining procedures. ${ }^{5,12-15}$

Though powerful, mesoscopic modeling faces a number of challenges. For example, soft potentials usually lead to simplified local liquid structure. This approximation does not affect the mesoscopic description of those properties that show scale separation. Such properties are governed on large scales by universal laws, ${ }^{16}$ encapsulating effects of microscopic details-including local liquid packing-into numerical prefactors of generic expressions. However, for many properties the concept of scale separation does not apply. Our work is concerned with one of such cases: the behavior of polymer selfentanglements or "knots".

Already for single (isolated) chains, simulations have shown that microscopic features largely affect knotting behavior. ${ }^{17-42}$ While short flexible ideal chains tend to form numerous ${ }^{17,22,24}$ highly localized knots, ${ }^{23}$ adding excluded volume interactions increases the chain lengths at which knots start to appear by about 2 orders of magnitude as, for example, expressed by surpassing a certain knotting probability. ${ }^{18,19,22,27}$ These knots are still (weakly) localized ${ }^{25-27}$ but considerably larger than in the ideal case. Overall, the effects of microscopic features can be very nontrivial. For example, recent studies ${ }^{43,44}$ have demonstrated that the probability of finding a randomly generated self-avoiding ring polymer in a knotted state changes nonmonotonously when the persistence length is increased, at fixed size of excluded volume and chain length. Intriguingly, for very long chains the change of probability has bimodal shape. ${ }^{44}$ Globular polymers and polymers confined to spheres are, on the other hand, heavily knotted, ${ }^{21,27}$ but their knots tend to be

Received: September 9, 2020

Revised: November 2, 2020

Published: November 18, 2020 
loose and delocalized ${ }^{25-27}$ and thus become a global property of the chain.

For multichain systems, modeling studies of knotting behavior are sparse. ${ }^{45-48}$ For the special case of polymer melts one can naively try to apply basic polymer physics. According to the Flory hypothesis, ${ }^{49}$ the effects from excluded volume interactions in melts are screened on scales exceeding the microscopic Edwards correlation length. Therefore, the conformations of polymers follow the statistics of ideal chains that have the same contour length and mean-squared end-toend distance as the chains in the melt. Recent studies ${ }^{50-52}$ have demonstrated that the Flory hypothesis is an approximation, which, nevertheless, becomes highly accurate for long chains. Therefore, one might expect that the properties of knots in melts of long chains and their equivalent ideal chains are similar.

Intriguingly, recent modeling studies ${ }^{48}$ have demonstrated that this assumption is too simplistic. In polymer melts knots appear significantly less frequently and are more loose when compared to equivalent isolated ideal chains. Apparently, even local excluded volume effects or, more generally, microscopic liquid structure can significantly influence the behavior of polymer knots. These observations lead to a basic question: Can mesoscopic models describe knots in polymer melts, given the absence of hard excluded volume and simplified local liquid structure?

Here, we address this fundamental question using a model that is typical for mesoscopic simulations of polymeric materials. The molecular architecture of long polymers is described through the worm-like chain (WLC) model. A generic soft repulsive potential captures nonbonded interactions between coarse-grained monomers. We define this potential through a simple particle-to-mesh scheme ${ }^{53-57}$ to deliberately obtain polymer liquids with very crude local structure. The soft model is parametrized to accurately reproduce mesoscopic structure and conformations of polymer melts that have been equilibrated in a previous study ${ }^{48}$ using a microscopic model. We directly compare the knotting properties in mesoscopic melts with their counterparts in the equivalent microscopically resolved samples.

We find that the ability of mesoscopic models to accurately describe knotting properties is crucially affected by the relationship between two length scales: the size of the excluded volume and the length scale characterizing the stiffness of the polymer chain. We qualitatively explain the trends of knotting behavior in mesoscopic and microscopic simulations benefiting from a free energy model of simple knots available in the literature. ${ }^{36,58}$

\section{KNOT DETECTION AND QUALITATIVE INSIGHTS FROM A FREE ENERGY MODEL}

Knots are only well-defined in closed loops and are categorized according to the minimum number of crossings in a projection onto a plane, for example, zero for the unknot or three for the trefoil $\left(3_{1}\right)$ knot. The concept can be extended to open chains by introducing an appropriate closure. The closure connects the termini of the chain in a well-defined manner ${ }^{20}$ before the knot type is determined with one of the available methods, for example, the calculation of Alexander polynomials ${ }^{59}$ which we use in this work. Here, we extend to infinity two straight lines, connecting the respective end points of a polymer chain and its center of mass. In this way, the closure originates at the first monomer, follows the first line outward, and connects to the second line far away from the polymer before returning to the last monomer along the second line. ${ }^{60}$ Knot sizes ${ }^{23,27}$ are determined by cleaving monomers from both ends until the knot type changes. ${ }^{27}$ Figure 1 presents a chain with a trefoil knot and clarifies the definition of the closure.

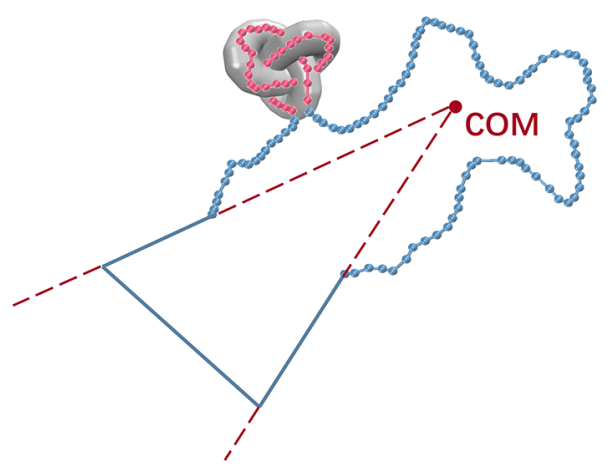

Figure 1. An open polymer chain with a trefoil knot. The knotted and unknotted parts are colored pink and blue, respectively. Two lines (red dashed lines) are determined based on the center of mass (COM) and two ends of the chain. The open chain is closed (blue solid lines) by connecting two points along these lines which are far from the chain. The formed trefoil knot is confined in a virtual maximum inflated tube (gray color).

To qualitatively understand the effect of local liquid structure on knots, it is useful to focus on the trefoil knot because it has the simplest possible topology. We consider the free energy $\operatorname{cost}^{36}$ - with respect to the unknotted state-to form a trefoil knot with contour length $N_{\text {knot }}$ in a chain with effective thickness $w$. This expression stems ${ }^{36}$ from a theory ${ }^{58}$ of knots in ideal chains (zero thickness). The thickness of the chain is determined by the size of the excluded volume and sets the characteristic length scale of local liquid structure. The free energy reads

$$
\beta F_{\text {knot }}=\frac{k_{1}}{2} \frac{1}{\tilde{N}_{\text {knot }}}+k_{2} \sqrt[3]{2} \frac{\tilde{N}_{\text {knot }}^{1 / 3}}{\left(1-\frac{p \tilde{w}}{\tilde{N}_{\text {knot }}}\right)^{2 / 3}}
$$

We use normalized variables $\tilde{N}_{\text {knot }}=N_{\text {knot }} / l_{\mathrm{K}}$ and $\tilde{w}=w / l_{\mathrm{K}}$, where $l_{\mathrm{K}}$ is the length of the Kuhn segment of the chain. The prefactors $1 / 2$ and $\sqrt[3]{2}$ make eq 1 equivalent to the original expression, ${ }^{36}$ casted in terms of $N_{\text {knot }} / l_{\mathrm{p}}$. Here $l_{\mathrm{p}}$ is the persistence length, and we assume $l_{\mathrm{K}} \simeq 2 l_{\mathrm{p}}$. The first term in $\beta F_{\text {knot }}$ penalizes ${ }^{36,58}$ chain bending and favors swollen knots. The second term expresses ${ }^{36,38}$ the entropic cost of constraining the chain into a virtual knotted tube and tends to shrink the knot. Figure 1 illustrates this virtual tube. The metastable size of the trefoil knot is given by the minimum of $\beta F_{\text {knot }} k_{1}$ and $k_{2}$ are positive phenomenological parameters. The constant $p$ is related to knot topology. ${ }^{36}$ Theoretically ${ }^{61} p$ $=12.4$, but it is preferable to treat $p$ also as a phenomenological parameter. ${ }^{36}$ Previous studies ${ }^{36,62}$ have tested $\beta F_{\text {knot }}$ against simulations of knots in isolated chains and determined $k_{1}, k_{2}$, and $p$. However, we can make some basic qualitative estimates without specifying these parameters; it is sufficient to know that they are positive quantities.

We notice that the convergence of $\beta F_{\text {knot }}$ to the free-energy landscape of trefoil knots in ideal chains is controlled by the magnitude of $\tilde{w}$. This parameter is small, even for large excluded volumes, if the polymer is sufficiently stiff, that is, has 
large $l_{\mathrm{K}}$. Consequently, for melts of stiff chains the knotting behavior should approach the universal limit of ideal chains, irrespective of local liquid structure. Therefore, we expect that the coarse description of liquid structure in mesoscopic models does not affect their ability to describe knots in polymer melts when chains are stiff.

Inspecting $\beta F_{\text {knot }}$ in eq 1 , we conclude that excluded volume always increases the entropic cost of confinement (second term). Therefore, the free energy cost for forming a trefoil knot with size $\tilde{N}_{\text {knot }}$ in a chain with excluded volume is always higher than for an ideal chain, with the same $l_{\mathrm{K}}$. Assuming that this trend holds also for more complex knots, $\beta F_{\text {knot }}$ suggests that the probability $P_{\mathrm{k}}$ to find a chain with excluded volume in a knotted state is smaller than in the case of an ideal chain. Moreover, according to $\beta F_{\text {knot }}$ for chains with excluded volume the entropic cost for forming a knot cannot be continuously diminished by decreasing the knot size. The large entropic cost of small knots shifts the minimum of $\beta F_{\text {knot }}$ to higher values compared to the ideal chain. In the Supporting Information we provide a formal proof based on minimization of $\beta F_{\text {knot. }}$ In other words, the most probable knot in a chain with excluded volume is larger than in its ideal chain counterpart. The difference between the two cases diminishes as the stiffness of the chain is increased, that is, as $\tilde{w} \rightarrow 0$.

\section{MICROSCOPICALLY RESOLVED REFERENCE MELTS}

The evaluation of the accuracy of mesoscopic models in capturing knotting properties of homopolymer melts requires reference data from systems described with microscopic detail. As a reference, we use the data on knotting properties of microscopically resolved melts that have been published in ref 48. When necessary, we additionally analyze the configurations of polymer melts equilibrated in that work to extract topological data that have not been included in the original publication. Below, we briefly discuss the main features of the microscopic model and summarize the characteristics of the reference samples.

The microscopically resolved melts occupy volume $V_{\text {mic }}$ and contain $n_{\text {mic }}$ linear bead-spring chains with $N_{\text {mic }}$ monomers each. The nonbonded interactions between the monomers are captured through the purely repulsive Weeks-ChandlerAndersen potential, where the length and the energy scales, $\sigma$ and $\epsilon$, are set to unity. In all cases the average bead density is set to $\rho=0.68 \sigma^{-3}$. The bonds between the monomers are described by harmonic springs, which are sufficiently stiff to ensure that the length of the bond remains practically fixed to $b_{\text {mic }}=0.967 \sigma$. To generate homopolymers with different stiffness, an angular potential $V(\theta)=B(1-\cos \theta)$ is introduced, where $\theta$ is the angle between two sequential bonds and $B$ is a stiffness parameter. The melts were equilibrated ${ }^{48}$ with molecular dynamics simulations, started from configurations prepared with a configuration-assembly method. ${ }^{63}$ Table 1 lists the polymerization degrees and stiffness parameters of all reference melts.

\section{MESOSCOPIC SIMULATIONS}

4.1. Model. Our mesoscopic description of homopolymer melts follows previous studies. ${ }^{57,64}$ Let the melt contain $n_{\mathrm{cg}}$ homopolymer chains in a volume $V_{\text {cg }}$. The homopolymers are represented by discrete worm-like chains (WLCs). Each of them has $N_{\mathrm{cg}}$ interaction centers, beads, connected by bonds
Table 1. Stiffness Parameter B, Polymerization Degree $N_{\text {mic }}$ Number of Kuhn Segments per Chain $N_{\mathrm{K}}$, and Square Root of Invariant Degree of Polymerization $\sqrt{\bar{N}}$ (See Eq 10) for the Different Reference Melts Considered in This Study

$\begin{array}{crrrr}\text { systems } & B & N_{\text {mic }} & N_{\mathrm{K}} & \sqrt{ } \bar{N} \\ \text { B0a } & 0 & 128 & 60.5 & 20.9 \\ \text { B0b } & & 256 & 121.4 & 29.8 \\ \text { B0c } & & 512 & 243.3 & 42.2 \\ \text { B0d } & & 1024 & 487.1 & 59.8 \\ \text { B2a } & 2 & 128 & 35.3 & 47.0 \\ \text { B2b } & & 256 & 70.8 & 66.8 \\ \text { B2c } & & 1024 & 141.9 & 94.8 \\ \text { B2d } & & 128 & 18.1 & 134.2 \\ \text { B4a } & 4 & 256 & 36.4 & 127.3 \\ \text { B4b } & & 512 & 73.0 & 181.1 \\ \text { B4c } & & 1024 & 146.1 & 256.9 \\ \text { B4d } & & & & 363.9\end{array}$

with fixed length $b_{\mathrm{cg}}$. The bonded potential of the WLC is given by

$$
\beta H_{\mathrm{b}}=-\epsilon_{0} \sum_{s=1}^{N_{\mathrm{cg}}-2} \mathbf{u}(s+1) \cdot \mathbf{u}(s)
$$

Here $\beta=1 / k_{\mathrm{B}} T, \mathbf{u}(s)$ is a unit vector along the sth bond, and $\epsilon_{0}$ is a positive parameter controlling the flexibility of the chain.

We define the nonbonded interactions through a particle-tomesh (PM) scheme. ${ }^{53-56}$ The volume occupied by the melt is discretized through a cubic lattice (grid) with spacing $\Delta L$. The grid enables the calculation of an instantaneous density "field" from the instantaneous coordinates of the $N_{\text {tot }}=n_{\mathrm{cg}} N_{\mathrm{cg}}$ beads, distributed in continuum space, using the relationship

$$
\hat{\rho}\left(\mathbf{c}_{m}\right)=\Delta L^{-3} \sum_{i=1}^{N_{\text {tot }}} \Pi\left(\mathbf{r}_{i}, \mathbf{c}_{m}\right)
$$

$\mathbf{r}_{i}$ is the position vector of the $i$ th bead, and $\mathbf{c}_{m}$ defines the location of the $m$ th node of the cell. The assignment function $\Pi\left(\mathbf{r}_{i}, \mathbf{c}_{m}\right)$ corresponds to a "zero-order" PM scheme ${ }^{3}$ and is defined as $\Pi=1$ when $-\Delta L / 2 \leq d_{\gamma}<\Delta L / 2$ and $\Pi=0$ otherwise. $d_{\gamma}$ are projections of $\delta \mathbf{r}_{i, m} \equiv \mathbf{r}_{i}-\mathbf{c}_{m}$ on the three Cartesian directions $\gamma=x, y, z$.

Based on the instantaneous densities, the nonbonded interactions are defined through the effective Hamiltonian:

$$
\beta H_{\mathrm{nb}}=\sum_{m=1}^{N_{\mathrm{cell}}} \frac{\Delta L^{3} \kappa}{2 \rho_{0}} \hat{\rho}\left(\mathbf{c}_{m}\right)^{2}
$$

where $\rho_{0}=N_{\text {tot }} / V_{\text {cg }}$ is the average density of the beads in the system. Because $\kappa$ is a positive coefficient, the effective Hamiltonian in eq 4 defines repulsive interactions.

The definition of $\beta \mathrm{H}_{\mathrm{nb}}$ through eq 4 facilitates numerical implementation (see subsection 4.3). However, to understand better the model and interpret its knotting properties, it is preferable to transform eq 4 by substituting for $\hat{\rho}\left(\mathbf{c}_{m}\right)$ the expression from eq 3 . Straightforward rearrangements lead ${ }^{55}$ to

$$
\beta H_{\mathrm{nb}}=\frac{\nu_{\kappa}}{2} \sum_{i=1}^{N_{\mathrm{tot}}} \sum_{j=1}^{N_{\mathrm{tot}}} \omega\left(\mathbf{r}_{i}, \mathbf{r}_{j}\right)
$$

where 


$$
\omega\left(\mathbf{r}_{i}, \mathbf{r}_{j}\right)=\sum_{m=1}^{N_{\text {cell }}} \Pi\left(\mathbf{r}_{i}, \mathbf{c}_{m}\right) \Pi\left(\mathbf{r}_{j}, \mathbf{c}_{m}\right), \quad \nu_{\kappa}=\frac{\kappa}{\rho_{0} \Delta L^{3}}
$$

Equations 5 and 6 convey an important message. They demonstrate that our mesoscopic model is a conventional particle-based model where non-bonded interactions between monomers are expressed through a pairwise non-bonded potential, $U\left(\mathbf{r}_{i}, \mathbf{r}_{j}\right)=\nu_{\kappa} \omega\left(\mathbf{r}_{i}, \mathbf{r}_{j}\right)$. Two monomers interact only when they are located in the same lattice cell, and the strength of their repulsion is controlled by $\nu_{\kappa}$. The pairwise potential in eq 6 is analogous to the well-known model of penetrable spheres ${ }^{65}$ but with an important difference: the PM-based $U\left(\mathbf{r}_{i}, \mathbf{r}_{j}\right)$ is translationally and rotationally noninvariant ${ }^{3}$ because it depends on the location of the interacting monomers with respect to the grid. The range of $U\left(\mathbf{r}_{i}, \mathbf{r}_{j}\right)$ varies between $\Delta L$ and $\sqrt{ } 3 \Delta L$ (depending on the position of the monomers in the cell); that is, in all cases the characteristic length scale of interactions is proportional to $\Delta L$.

4.2. Parametrization. We now must determine the parameters of the mesoscopic model, $N_{\mathrm{cg}}, b_{\mathrm{cg}}, \epsilon_{0}, n_{\mathrm{cg}} / V_{\mathrm{cg}}$ (chain density), $\Delta L$, and $\kappa$, such that the coarse-grained homopolymer melts represent the reference systems. To make progress, we recall that within the (approximate ${ }^{51}$ ) Flory hypothesis ${ }^{49}$ long flexible chains in polymer melts follow the statistics of ideal random walks and can be "assigned" an equivalent Kuhn chain. The latter is a freely jointed chain composed of $N_{\mathrm{K}}$ rigid segments, with length $l_{\mathrm{K}}$ each. Because $l_{\mathrm{K}}$ is a characteristic length along the polymer-chain contour at which the chain can be considered as "straight", it sets the scale at which the formation of knots becomes unlikely. Therefore, one of the requirements for mesoscopically and microscopically resolved melts to have the same knotting properties is that they map on the same Kuhn chains; that is, they have the same $l_{\mathrm{K}}$ and $N_{\mathrm{K}}$. We emphasize that this requirement is only a necessary but not sufficient ${ }^{48}$ condition. Clarifying additional conditions for having the same behavior of knots is among the objectives of our study.

The parameters of the equivalent Kuhn chain are expressed through the contour length $L$ and mean-square end-to-end distance $\left\langle R_{\mathrm{e}}{ }^{2}\right\rangle$ of an ideal polymer chain as ${ }^{66} l_{\mathrm{K}}=\left\langle R_{\mathrm{e}}{ }^{2}\right\rangle / L$ and $N_{\mathrm{K}}=L^{2} /\left\langle R_{\mathrm{e}}{ }^{2}\right\rangle$. Hence, mesoscopically and microscopically resolved melts map on identical Kuhn chains only when they have the same $L$ and $\left\langle R_{\mathrm{e}}{ }^{2}\right\rangle$. The contour length of the WLC is given by

$$
L=\left(N_{\mathrm{cg}}-1\right) b_{\mathrm{cg}}
$$

Assuming that the conformations of WLC chains in the melt obey ideal random walk statistics, their mean-square end-toend distance is given by the well known expression ${ }^{67}$

$$
\left\langle R_{\mathrm{e}}{ }^{2}\right\rangle=\left(N_{\mathrm{cg}}-1\right) b_{\mathrm{cg}}{ }^{2}\left[\frac{1+G\left(\epsilon_{0}\right)}{1-G\left(\epsilon_{0}\right)}-\frac{2 G\left(\epsilon_{0}\right)}{N_{\mathrm{cg}}-1} \frac{1-G\left(\epsilon_{0}\right)^{N_{\mathrm{cg}}-1}}{\left(1-G\left(\epsilon_{0}\right)\right)^{2}}\right]
$$

where $G\left(\epsilon_{0}\right)$ quantifies $^{68}$ the angular correlation between two successive bonds. If $\theta_{0}$ is the instantaneous angle formed by these bonds, then

$$
G\left(\epsilon_{0}\right) \equiv\left\langle\cos \theta_{0}\right\rangle=\frac{1-\epsilon_{0}^{-1}+\mathrm{e}^{-2 \epsilon_{0}}\left(1+\epsilon_{0}^{-1}\right)}{1-\mathrm{e}^{-2 \epsilon_{0}}}
$$

For the reference systems the contour length follows from the geometry of the bead-spring chain as $L=\left(N_{\text {mic }}-1\right) b_{\text {mid }}$ while $\left\langle R_{\mathrm{e}}^{2}\right\rangle$ is calculated as an average over all chains in all equilibrated samples of the given melt. Substituting into the left-hand side of eqs 7 and 8 the $L$ and $\left\langle R_{\mathrm{e}}{ }^{2}\right\rangle$ of the reference systems, we obtain two constraints on three parameters $N_{\text {cg }}$, $b_{\text {cg }}$, and $\epsilon_{0}$. Because the system of eqs 7 and 8 is underconstrained, there is an infinite number of different WLC models ${ }^{57}$ that map on the same Kuhn chain (have the same $L$ and $\left.\left\langle R_{\mathrm{e}}{ }^{2}\right\rangle\right)$. Therefore, we consider $N_{\mathrm{cg}}$ as a free parameter but with some restrictions. Whereas the discretization of the WLC should be finer than or (at least) comparable to the Kuhn chain, i.e., $N_{\mathrm{cg}} \geq N_{\mathrm{K}}, N_{\mathrm{cg}}$ cannot be too large for reasons of computational efficiency. As a compromise we set $N_{\mathrm{cg}}=2 N_{\mathrm{K}}$. For each reference melt, we summarize the $N_{\mathrm{K}}$ in the equivalent Kuhn chains in Table 1 (extracted from their $L$ and $\left.\left\langle R_{\mathrm{e}}{ }^{2}\right\rangle\right)$. In practice, the conformations of WLC in the coarse-grained homopolymer melts are not determined by $\epsilon_{0}{ }^{69}$ only, but are also affected by nonbonded interactions. Consequently, for given $N_{\mathrm{cg}}$ and $b_{\mathrm{cg}}$, we use eq 8 only to extract an initial estimate for $\epsilon_{0}$. This estimate is refined with iterative simulations of the entire mesoscopic homopolymer melt to ensure that the target value $\left\langle R_{\mathrm{e}}{ }^{2}\right\rangle$ is reproduced in the ensemble of interacting WLC.

In addition to the length scales $L$ and $\left\langle R_{\mathrm{e}}{ }^{2}\right\rangle$, the mesoscopic model should reproduce another key property of the reference homopolymer melts-the invariant degree of polymerization $\bar{N}$. It is defined as

$$
\sqrt{\bar{N}}=\frac{\rho}{N_{\text {mic }}}\left\langle R_{\mathrm{e}}^{2}\right\rangle^{3 / 2}=\frac{n_{\text {mic }}}{V_{\text {mic }}}\left\langle R_{\mathrm{e}}^{2}\right\rangle^{3 / 2}
$$

$\sqrt{\bar{N}}$ quantifies the number of molecules threading through the characteristic volume of a test chain. Conserving $\bar{N}$ is crucial because it determines the mesoscale structure of the polymer liquid $^{16,70}$ in a universal way. Importantly, the magnitude of deviations from the Flory hypothesis is controlled, to first order, $^{50-52}$ by $\sim 1 / \sqrt{\bar{N}}$. In other words, $1 / \sqrt{\bar{N}}$ is the parameter that determines how well polymer conformations in melts can be approximated by ideal chains. For all reference systems, we list the $\sqrt{\bar{N}}$ in Table 1 .

Based on eq 10 , to conserve $\sqrt{\bar{N}}$, the mesoscopic model must reproduce the chain density of the reference melts (because $\left\langle R_{\mathrm{e}}{ }^{2}\right\rangle$ is also a conserved quantity):

$$
\frac{n_{\mathrm{cg}}}{V_{\mathrm{cg}}}=\frac{n_{\mathrm{mic}}}{V_{\mathrm{mic}}}
$$

Having clarified how generic properties of polymer melts determine some of our parameters, we turn to the modelspecific, local, liquid structure and consider the quantity

$$
\eta=\rho_{0} \Delta L^{3}=\frac{n_{\mathrm{cg}} N_{\mathrm{cg}}}{V_{\mathrm{cg}}} \Delta L^{3}
$$

Because the lattice spacing $\Delta L$ sets the range of the repulsion, $\eta$ is a monomer packing fraction-a well-known parameter in mesoscopic models with standard (translationally and rotationally invariant) pairwise potentials. We vary the local liquid structure in the mesoscopic simulations, by modeling melts with weak, $\eta<1$, and strong, $\eta>1$, overlap of monomers. To realize the desired $\eta$, we modify $\Delta L$ under the constraint that $\Delta L<l_{\mathrm{K}}$. This condition ensures that the model-specific length scale of interaction range is separated from the generic length scale of chain rigidity. 
Varying the softness of the repulsive potential is an alternative way for modifying local liquid structure. We change $\kappa$ such that the strength of monomer-monomer repulsion $\nu_{\kappa}$ varies from a fraction of $k_{\mathrm{B}} T$ to a few tens of $k_{\mathrm{B}} T$.

4.3. Monte Carlo Sampling. The configurational space is sampled with PM Monte Carlo (MC) simulations, described in several previous studies. ${ }^{53,55,57,64}$ In short, our simulations are performed in the canonical ensemble and consider cubic samples of melts with periodic boundary conditions (PBC). The edge of the cube equals $2 \sqrt{\left\langle R_{\mathrm{e}}{ }^{2}\right\rangle}$; this empirical choice is widely accepted as the minimum requirement for avoiding finite-system size effects in disordered melts.

We employ two standard MC moves: the slithering snake ${ }^{71}$ (reptation) and the crankshaft move ${ }^{72}$ (also known as "flip" move $\left.{ }^{73}\right)$. In the reptation move, a homopolymer chain is randomly selected. One segment is deleted from a randomly chosen chain end and is attached to the other end. In the crankshaft move, the rotation angle ${ }^{72,73}$ is randomly chosen from the interval $[0,2 \pi]$. The acceptance criterion for reptation and flip moves is formulated in a similar way. From the coordinates of the displaced bead in its new and old positions, $\mathbf{r}^{\text {(new) }}$ and $\mathbf{r}^{\text {(old) }}$, we calculate the difference in the bonded energies $\Delta\left(\beta H_{\mathrm{b}}\right)$ caused by the MC move. Furthermore, $\mathbf{r}^{\text {(new) }}$ and $\mathbf{r}^{\text {(old) }}$ are used to identify the cells that are influenced by the move and calculate the new densities, $\rho^{\text {new }}\left(\mathbf{c}_{m}\right)$, in these cells. For both MC moves used in this work, the amount of affected cells is either zero, if the bead lands in the same cell as before, or two. Taking into account the bead densities in the affected cells before the move, $\rho^{\text {old }}\left(\mathbf{c}_{m}\right)$, it is straightforward to calculate the change in the nonbonded energy $\Delta\left(\beta H_{\mathrm{nb}}\right)$, based on eq 4 . The move is accepted with probability $p_{\text {acc }}=\min \left\{1, \exp \left[-\Delta\left(\beta H_{\mathrm{b}}\right)-\right.\right.$ $\left.\left.\Delta\left(\beta H_{\mathrm{nb}}\right)\right]\right\}$.

\section{STRUCTURE AND CONFORMATIONS}

In this section we investigate basic structural and conformational properties of our mesoscopic melts. These properties are required for (i) quantifying the accuracy with which our soft model reproduces the mesoscopic features of the reference systems and (ii) interpretation of the behavior of knots.

We first demonstrate the simplified local liquid structure in the mesoscopic model by presenting monomer-monomer radial distribution functions $g(r)$ in Figure 2a. The distance is scaled by the bond length of the WLC so that the "spike" of the $g(r)$, due to WLC connectivity, is always at $r / b_{\mathrm{cg}}=1$. We consider a representative family of melts whose parameters have been adjusted to reproduce the mesoscopic propertiesKuhn chain and $\sqrt{\bar{N}}$ - of the B2c reference melt. However, we vary the local structure of these melts by changing the strength of the monomer-monomer repulsion $\nu_{\kappa}$ at fixed $\eta=1$. We quantify the depth of the monomer-monomer correlation hole in the inset of Figure 2 a by presenting $g(r \simeq 0)$ as a function of $\nu_{\kappa}$. As expected, $g(r \simeq 0)$ decreases with the increase of $\nu_{\kappa}$ but saturates to a small value, instead of converging towards zero in the limit of hard repulsions $\left(\nu_{\kappa} \rightarrow\right.$ $+\infty)$. We conclude that we cannot completely eliminate overlaps between monomers by increasing their repulsion, in contrast to models with traditional translationally invariant potentials.

This peculiar behavior of monomer packing at strong repulsions stems from the zero-order PM scheme. We demonstrate this by rewriting the radial distribution function as
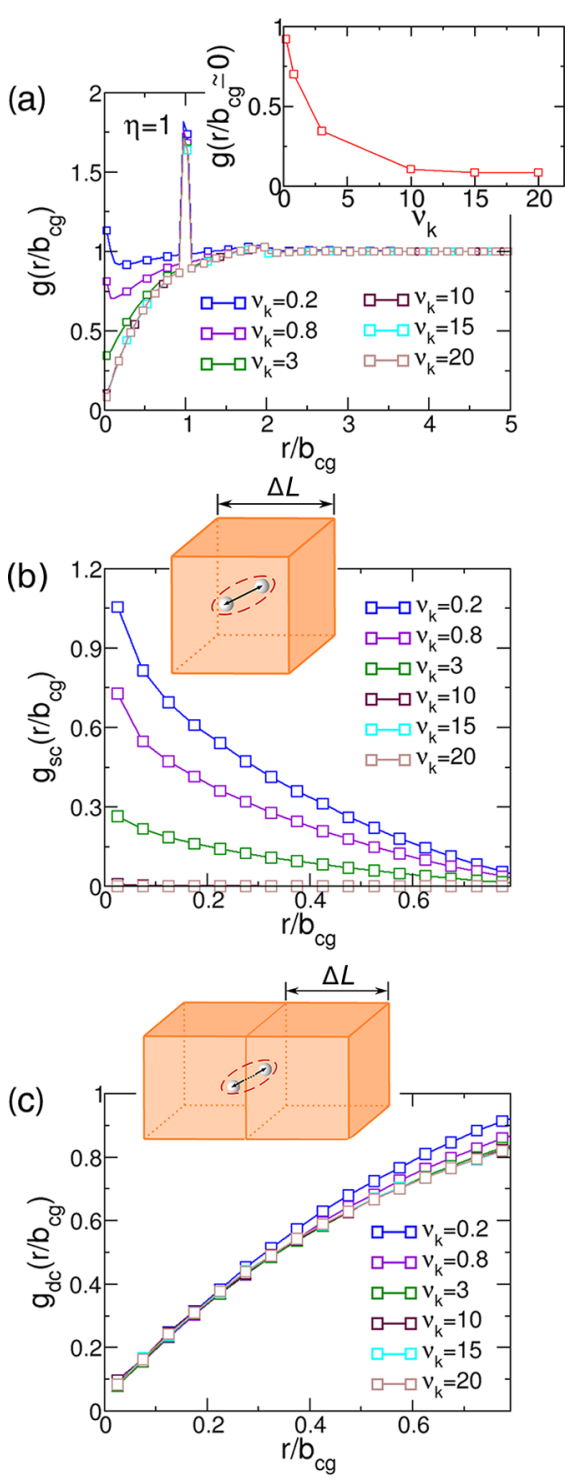

Figure 2. Radial distribution functions in mesoscopic melts representing the $\mathrm{B} 2 \mathrm{c}$ reference system. The melts have the same monomer packing fraction $\eta=1$ but different strength of repulsive interactions $\nu_{k}$, as indicated in the legends. The main figure of (a) shows the total $g(r)$ of CG beads, and the inset demonstrates $g(r \simeq 0)$ as a function of $\nu_{\kappa^{*}}$ (b) and (c) show the partial radial distribution functions $g_{\mathrm{sc}}(r)$ and $g_{\mathrm{dc}}(r)$ calculated, respectively, for pairs of monomers found in the same cell and two different neighboring cells. The sketches in the insets of (b) and (c) illustrate these two different types of monomer pairs. In all plots, the distance $r$ is scaled by the bond length $b_{\mathrm{cg}}$ of the WLC.

$$
g(r)=g_{\mathrm{sc}}(r)+g_{\mathrm{dc}}(r)
$$

where

$$
g_{\mathrm{sc}}(r)=\frac{1}{A}\left\langle\sum_{i}^{N_{\mathrm{tot}}} \sum_{j, j \neq i}^{N_{\mathrm{tot}}} \delta\left(r_{i j}-r\right) \omega\left(\mathbf{r}_{i}, \mathbf{r}_{j}\right)\right\rangle
$$

and

$$
g_{\mathrm{dc}}(r)=\frac{1}{A}\left\langle\sum_{i}^{N_{\text {tot }}} \sum_{j, j \neq i}^{N_{\text {tot }}} \delta\left(r_{i j}-r\right)\left(1-\omega\left(\mathbf{r}_{i}, \mathbf{r}_{j}\right)\right)\right\rangle
$$


Here $r_{i j}=\left|\mathbf{r}_{i}-\mathbf{r}_{j}\right|$ and $A=1 / 4 \pi r^{2} N_{\text {to }} \rho_{0}$. Equations 14 and 15 demonstrate that $g_{\mathrm{sc}}(r)$ and $g_{\mathrm{dc}}(r)$ quantify the contribution to $g(r)$ from pairs of particles that are found in the same and different cells, respectively.

Figures $2 \mathrm{~b}$ and $2 \mathrm{c}$ present the components $g_{\mathrm{sc}}(r)$ and $g_{\mathrm{dc}}(r)$ of $g(r)$ from Figure $2 \mathrm{a}$, focusing on the region of small distances. From the plots of $g_{\mathrm{sc}}(r)$ we conclude that there are less and less pairs of monomers found in the same cell (cf. sketch in the inset of Figure $2 \mathrm{~b}$ ) as $\nu_{\kappa}$ increases. In contrast, the saturation of the plots of $g_{\mathrm{dc}}(r)$ demonstrates that the contribution to $g(r)$ from pairs of monomers found on different sides of the boundary of neighboring lattice cells (cf. sketch in the inset of Figure 2c) becomes constant at large $\nu_{\kappa}$. Such monomers can be arbitrarily close because they do not interact with each other. In passing, we mention that the amount of pairs of such close monomers, presumably, will be reduced in higher order PM schemes ${ }^{3,56}$ where the density of a monomer is smeared over several cells.

Figure 3 presents $g(r)$ for mesoscopic melts that are again equivalent to the B2c system. Now, however, we set $\nu_{\kappa}=3$ and

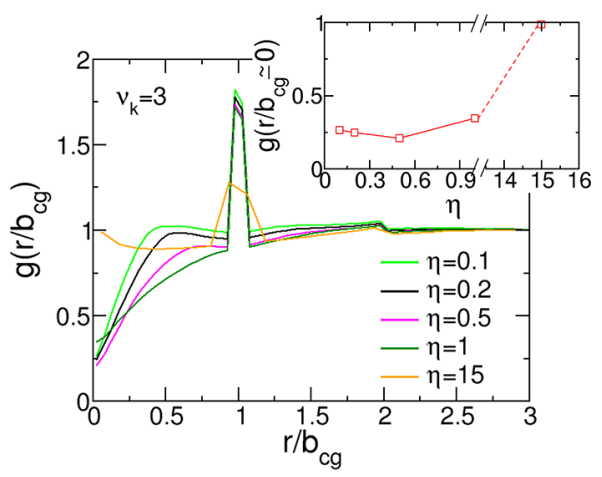

Figure 3. Radial distribution functions in mesoscopic melts representing the $\mathrm{B} 2 \mathrm{c}$ reference system. The melts have the same strength of repulsive interactions $\nu_{\kappa}=3$ but different monomer packing fractions $\eta$, as indicated in the legends. In all plots, the distance $r$ is scaled by the bond length $b_{c g}$ of the WLC.

vary $\eta$ by adjusting $\Delta L$. Specifically, $\Delta L$ varies from $0.37 b_{\mathrm{cg}}(\eta$ $=0.1)$ until $1.98 b_{\mathrm{cg}}(\eta=15)$. The inset of Figure 3 presents $g(r$ $\simeq 0$ ) as a function of $\eta$. For $\eta \leq 1$ we observe a distinct monomer-monomer correlation hole because at low $\eta$ it is possible to achieve energetically favorable nonoverlapping monomer arrangements when cells are occupied by single monomers. The width of the correlation hole is proportional to the range of the repulsion $\Delta L$, similarly to models with translationally invariant potentials. However, the behavior of the depth of the correlation hole is more complex: for $\eta \leq 1$, $g(r \simeq 0)$ first decreases as $\eta$ becomes smaller but rises again for $\eta \leq 0.5$. This nonmonotonicity has the same origin as the nontrivial effects of $\nu_{\kappa}$ on monomer overlap. Decomposing the $g(r)$ from Figure 3 into $g_{\mathrm{sc}}(r)$ and $g_{\mathrm{dc}}(r)$ (see Supporting Information) demonstrates that the contribution of monomer pairs to $g(r)$ found in the same cell decreases as $\eta$ becomes smaller. In contrast, the contribution from pairs of monomers found on different sides of the boundary of adjacent lattice cells increases. Eventually, at small $\eta, g_{\mathrm{dc}}(r)$ outweighs $g_{\mathrm{sc}}(r)$ so that $g(r \simeq 0)$ grows again.

In contrast to monomer-monomer packing, the liquid structure on the scale of the entire WLC is almost independent from the details of interactions, consistent with the ideas of universality. On this scale, the mesoscopic model reproduces the liquid structure of the reference, microscopically resolved, melts. We provide a demonstration in Figures $4 a$ and $4 b$ by
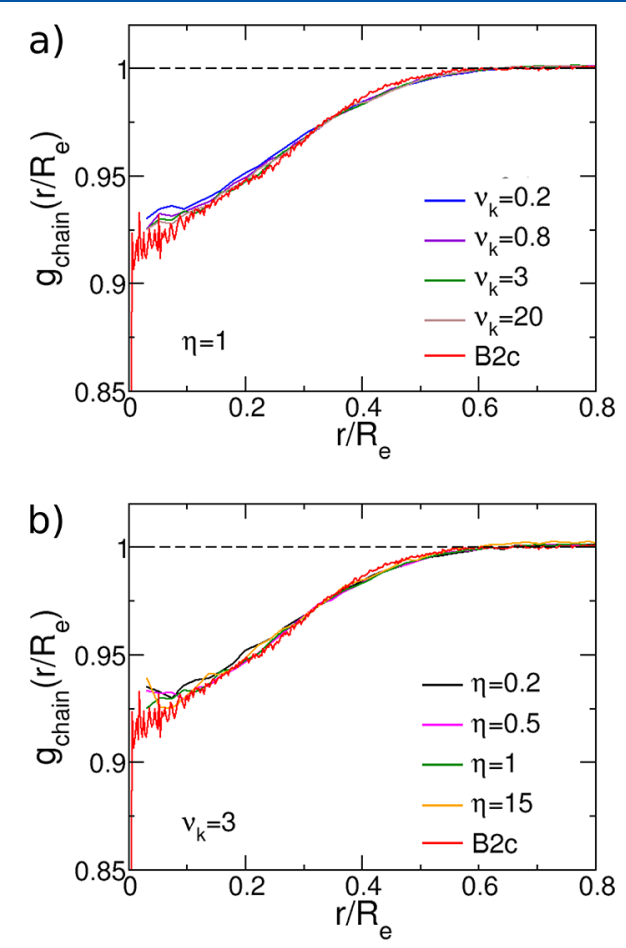

Figure 4. Radial distribution functions $g\left(r / R_{\mathrm{e}}\right)$ of center of mass of polymer chains calculated in mesoscopic melts representing the B2c reference melt. The mesoscopic simulations correspond to (a) different $\nu_{\kappa}$ but fixed $\eta=1$ and (b) different $\eta$ but fixed $\nu_{\kappa}=3$. The solid red line presents the $g\left(r / R_{\mathrm{e}}\right)$ calculated in the B2c reference melt. The distance $r$ is scaled by the root-mean-square end-to-end distance $R_{\mathrm{e}}$ of the polymer.

presenting the radial distribution function $g_{\text {chain }}(r)$ of the centers of mass of the chains in the mesoscopic melts that have been considered in Figures 2 and 3. The distance $r$ is scaled by the root-mean-square end-to-end distance, $R_{\mathrm{e}}=\sqrt{\left\langle R_{\mathrm{e}}{ }^{2}\right\rangle}$ of the polymer. The plots follow closely the $g_{\text {chain }}(r)$ in the reference $\mathrm{B} 2 \mathrm{c}$ melt (the deviations are at most $2 \%$ ).

It is crucial to examine explicit quantifiers of polymer conformations because they directly correlate with knotting properties. The internal distance plot is considered ${ }^{63,74}$ as one of the most sensitive quantifiers of polymer conformations. For our melts, it is defined as $C_{\alpha, m}(s) \equiv\left\langle R_{\alpha, m}{ }^{2}(s) / s\right\rangle$, where $\left\langle R_{\alpha, m}{ }^{2}(s)\right\rangle$ is the mean-square distance of two intramolecular repeat units separated by $s$ bonds along the contour of the WLC or the bead-spring chain ( $s$ is also known as the "chemical distance"). The subscript $\alpha$ indicates whether we are considering a mesoscopic or a reference melt, $\alpha=\mathrm{cg}$ and mic, respectively. The subscript $m$ denotes a specific system listed in Table 1, e.g., $m=\mathrm{B} 2 \mathrm{c}$. Figure 5a presents the $C_{\mathrm{cg}, \mathrm{B} 2 \mathrm{c}}(s)$ for a few representative mesoscopic analogues of the B2c melt and compares them with $C_{\text {mic,B2c }}(s)$ from the reference system. Although the mesoscopic WLC and bead-spring chains have different architecture and discretization (number of monomers), we can compare their internal distance plots on a common graph using a rescaled chemical distance $\tilde{s}=s b_{\alpha} / l_{\mathrm{K}}$.

We quantify the difference between $C_{\mathrm{cg}, m}(\tilde{s})$ and $C_{\text {mic }, m}(\tilde{s})$ through their relative deviation: 

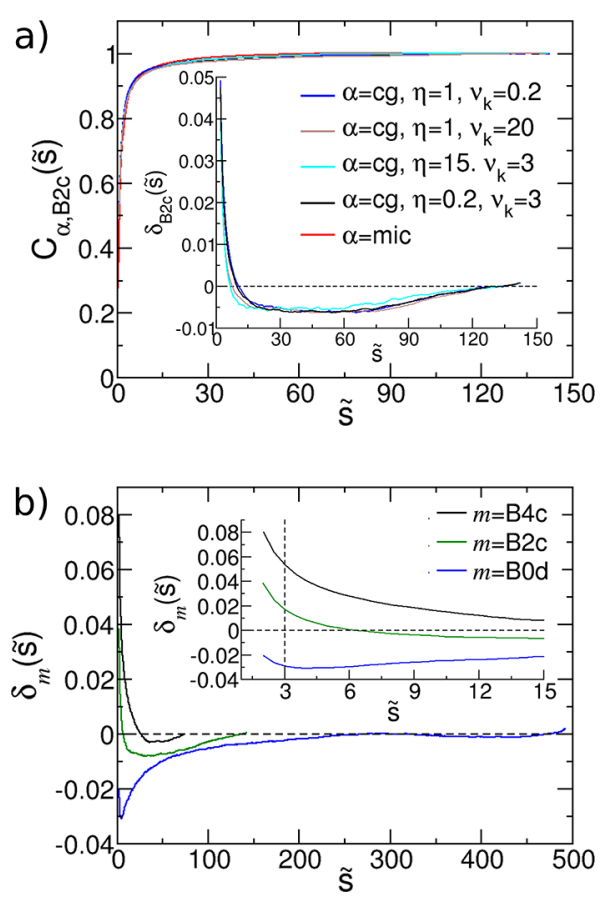

Figure 5. (a) Internal distance plot $C_{\mathrm{cg}, \mathrm{B} 2 \mathrm{c}}(\tilde{s})$ calculated as a function of rescaled chemical distance $\tilde{s}$ in mesoscopic polymer melts describing the $\mathrm{B} 2 \mathrm{c}$ reference melt, for representative sets of parameters. The red solid line shows the internal distance plot $C_{\text {mic,B2c }}(\tilde{s})$ of the reference $\mathrm{B} 2 \mathrm{c}$ melt. The inset presents the relative deviation $\delta_{\mathrm{B} 2 \mathrm{c}}(\tilde{s})$ between the internal distance plots in the mesoscopic melts and the reference B2c system. (b) Relative deviations $\delta_{m}(\tilde{s})$ of internal distance plots in mesoscopic melts, mapped from reference melts with different stiffness, from their counterparts in these reference melts. The inset focuses on the region of small $\tilde{s}$, where the vertical dashed line marks the relative deviation at the rescaled chemical distance corresponding to three Kuhn segments, $\tilde{s}=3$.

$$
\delta_{m}(\tilde{s})=\frac{C_{\mathrm{cg}, m}(\tilde{s})-C_{\mathrm{mic}, m}(\tilde{s})}{C_{\mathrm{mic}, m}(\tilde{s})}
$$

The relative deviations $\delta_{\mathrm{B} 2 \mathrm{c}}$ shown in the inset of Figure 5a, demonstrate that for chemical distances that are equal to or larger than three Kuhn segments, $\tilde{s} \geq 3$, the differences between the internal distance plots in the mesoscopic melts and the reference $\mathrm{B} 2 \mathrm{c}$ system are already reduced to $3 \%$ at most. Such conformational deviations are considered small in other areas of polymer modeling, e.g., hierarchical backmapping. ${ }^{14,75}$ The conformational deviations are somewhat larger for mesoscopic melts representing the more flexible, $B=$ 0 , and stiffer, $B=4$, reference systems. Figure 5 b presents $\delta_{m}(\tilde{s})$ for the mesoscopic analogues of B0d and B4c reference melts. The simulations were performed with $\nu_{\kappa}=3$ and $\eta=1$. To facilitate comparison, we add $\delta_{\mathrm{B} 2 \mathrm{c}}(\tilde{s})$ (extracted from simulations also performed with $\nu_{\kappa}=3$ and $\left.\eta=1\right)$. The deviations at $\tilde{s}=3$ correspond to about $3 \%$ for B0d and $5 \%$ for $\mathrm{B} 4 \mathrm{c}$ melts; the inset presents enlarged plots, where the vertical dashed line marks $\tilde{s}=3$.

Even for the smallest $\nu_{\kappa}$ and $\eta$ considered in this study, the mesoscopic chains reproduce ${ }^{55}$ subtle features of conformational statistics expected for melts with interacting polymer chains. To clearly demonstrate this point, we calculate the standard decay of bond-bond correlations $P(s)=\left\langle\mathbf{u}\left(s_{0}\right) \mathbf{u}\left(s_{0}+\right.\right.$ $s)\rangle$. Here $\mathbf{u}\left(s_{0}\right)$ and $\mathbf{u}\left(s_{0}+s\right)$ are vectors oriented along the $s_{0}$ and the $s_{0}+s$ bond. Recent studies ${ }^{50,51}$ have demonstrated that in polymer melts $P(s)$ does not decay exponentially at large $s$ (as predicted by the Flory hypothesis) but follows the scaling $P(s) \sim s^{-3 / 2}$. Figure 6 presents the $P(\tilde{s})$ calculated for all

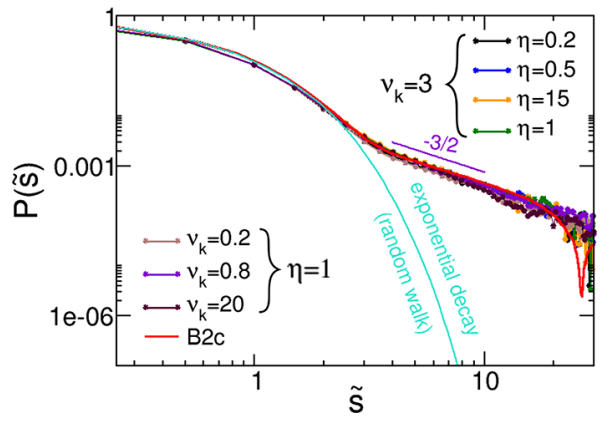

Figure 6. Decay of bond-bond correlations along the chain contour in mesoscopic melts describing the B2c reference system. The results obtained for various representative choices of monomer-monomer repulsion strength $\nu_{\kappa}$ and monomer packing fraction $\eta$ are compared with the decay of bond-bond correlations in the reference B2c melt (red solid line). The light blue solid line describes the exponential decay of bond-bond correlations in the equivalent ideal Kuhn chain. The purple solid line shows the power-law decay $P(s) \sim s^{-3 / 2}$, expected in polymer melts.

equivalent mesoscopic representations of the B2c melt together with the data from the reference system by using the rescaled chemical distance $\tilde{s}$. For the region $0<\tilde{s}<10$, where the statistics are fairly good, we observe that the decay of bond-bond correlations reproduces the correct power law and follows closely the reference data.

\section{PROPERTIES OF KNOTS IN THE MESOSCOPIC MODEL}

We just saw that the local liquid structure in our mesoscopic melts is very coarse. Moreover, this structure varies across families of melts that are mesoscopically equivalent to the same reference system. In contrast, mesoscopic liquid packing and conformational properties of reference systems are well reproduced. Therefore, our mesoscopic melts are indeed well suited for verifying the qualitative predictions on knotting behavior, obtained from the simple free energy model.

First we consider melts with intermediate chain stiffness: the B2 systems. Using the predictions obtained in section 2 from $\beta F_{\text {knot }}$ we can easily estimate whether local monomer packing is important for the knotting properties of these systems. We consider the configurations of the representative B2c melt and calculate the probability $P_{\mathrm{k}}$ that a randomly selected chain is found in a knotted state. The knotting probability $P_{\mathrm{k}}$ is presented in Figure $7 \mathrm{a}$ (horizontal red line) and is about 3 times smaller than the $P_{\mathrm{k}}$ (horizontal light blue line) in ideal chains that map on the same Kuhn chain as the B2c melt. The large difference between the two knotting probabilities indicates that the polymers in B2 melts are still rather flexible. We can expect that the knotting behavior in B2 melts is dominated by excluded volume effects and cannot be accurately reproduced by the mesoscopic model.

The analysis of mesoscopic simulations confirms this expectation. Figure $7 \mathrm{a}$ presents $P_{\mathrm{k}}$ as a function of $\nu_{\kappa}$ calculated in several mesoscopic melts representing the B2c system (in all these cases $\eta=1$ ). All calculated $P_{\mathrm{k}}$ are between the knotting probabilities of the reference melt and its equivalent ideal 

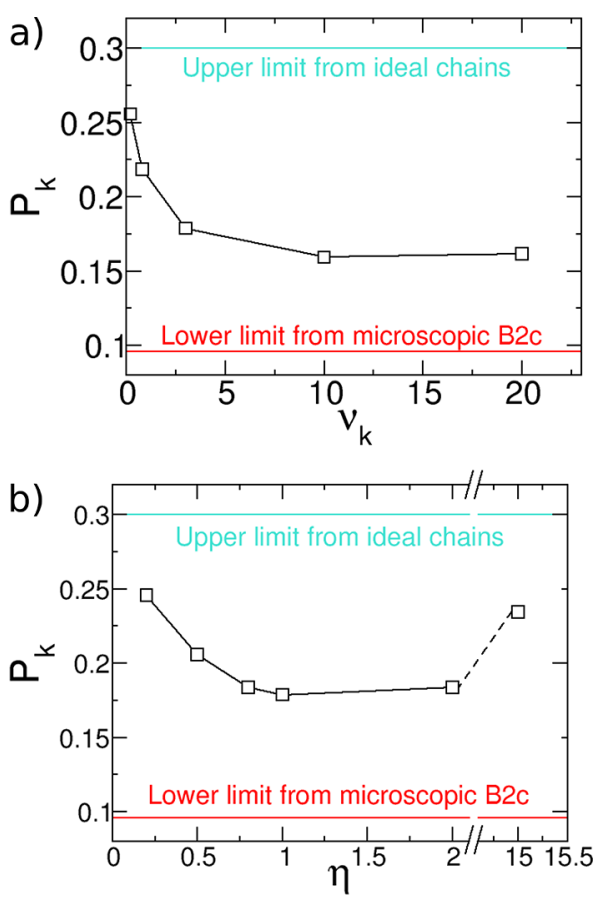

Figure 7. Effect of (a) repulsion strength $\nu_{\mathrm{k}}$ and (b) average number of overlapping particles $\eta$ on the knotting probability $P_{\mathrm{k}}$ of the mesoscopic melts. All mesoscopic melts are equivalent to the B2c system. The knotting probability in the reference B2c melt and in ideal Kuhn chains with same $N_{\mathrm{K}}$ are shown with red and light blue solid lines, respectively.

Kuhn chain. $P_{\mathrm{k}}$ decreases sharply as the repulsion becomes stronger but finally converges to a plateau that is about $50 \%$ larger than the $P_{\mathrm{k}}$ in the reference B2c melt. This behavior stems from insufficiently hard excluded volume, even at strong repulsions, because of the zero-order PM scheme (cf. Figure 2 and related discussion). Figure $8 \mathrm{a}$ shows a representative

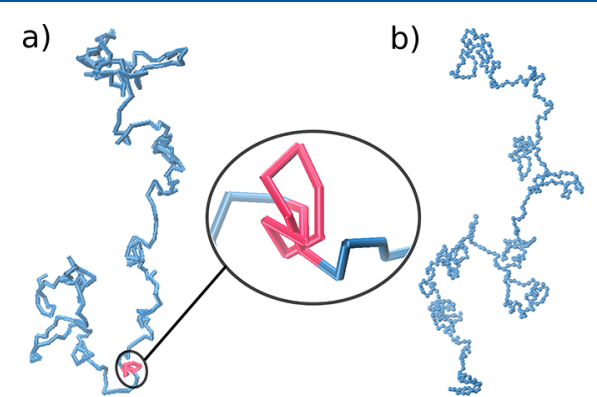

Figure 8. Representative chain conformations from polymer melts. Part a shows a knotted WLC in a mesoscopic melt representing the B2c reference melt. The chain forms a very small trefoil knot with contour length $\tilde{N}_{\text {knot }}=4.5$. The trefoil knot is shown with pink color. Part $b$ presents an unknotted microscopically resolved chain in the reference $\mathrm{B} 2 \mathrm{c}$ melt.

snapshot of a small knot formed on a chain in the mesoscopic B2c melt. Despite the presence of very small knots, the appearance of this mesoscopic chain on large scales is similar to a representative microscopic B2c chain (see Figure $8 b$ ).

To complement Figure $7 \mathrm{a}$, we present $P_{\mathrm{k}}$ in Figure $7 \mathrm{~b}$ as a function of $\eta$ for several mesoscopic melts representing the B2c melt (in all these cases $\nu_{\kappa}=3$ ). The dependence of $P_{\mathrm{k}}$ on $\eta$ is nonmonotonous. $P_{\mathrm{k}}$ is large at $\eta \ll 1$, decreases as $\eta$ increases, reaches a broad minimum at $\eta \simeq 1$, and grows again (presumably towards the limit of the ideal chain). The reduction of $P_{\mathrm{k}}$, as $\eta$ becomes larger in the region $\eta \leq 1$, is caused by the increase of the size of excluded volume (see width of correlation hole in $g(r)$ in Figure 3 ) and is consistent with qualitative expectations from eq 1 . For $\eta \gg 1, P_{\mathrm{k}}$ increases again because for such strong overlaps a test monomer finds itself in a uniform "background field" of other monomers. There is no energetic penalty for monomer-monomer overlap, and the excluded volume effectively tends to zero.

The effect of polymer stiffness on the accuracy of mesoscopic models in describing knotting properties is best illustrated by comparing trefoil knots in the mesoscopic and reference melts. In Figures $9 a, 9 b$, and $9 c$ we present the distribution of sizes of trefoil knots $P\left(\tilde{N}_{\text {knot }}\right)$ in the mesoscopic melts describing B0d, B2c, and B4c reference systems, respectively (the simulations were performed with $\nu_{\kappa}=3$ and $\eta=1)$. In the same panels, we also present the $P\left(\tilde{N}_{\text {knot }}\right)$ in reference melts and in their equivalent ideal Kuhn chains. The size of the trefoil knots $\tilde{N}_{\text {knot }}$ is defined according to section 2 .
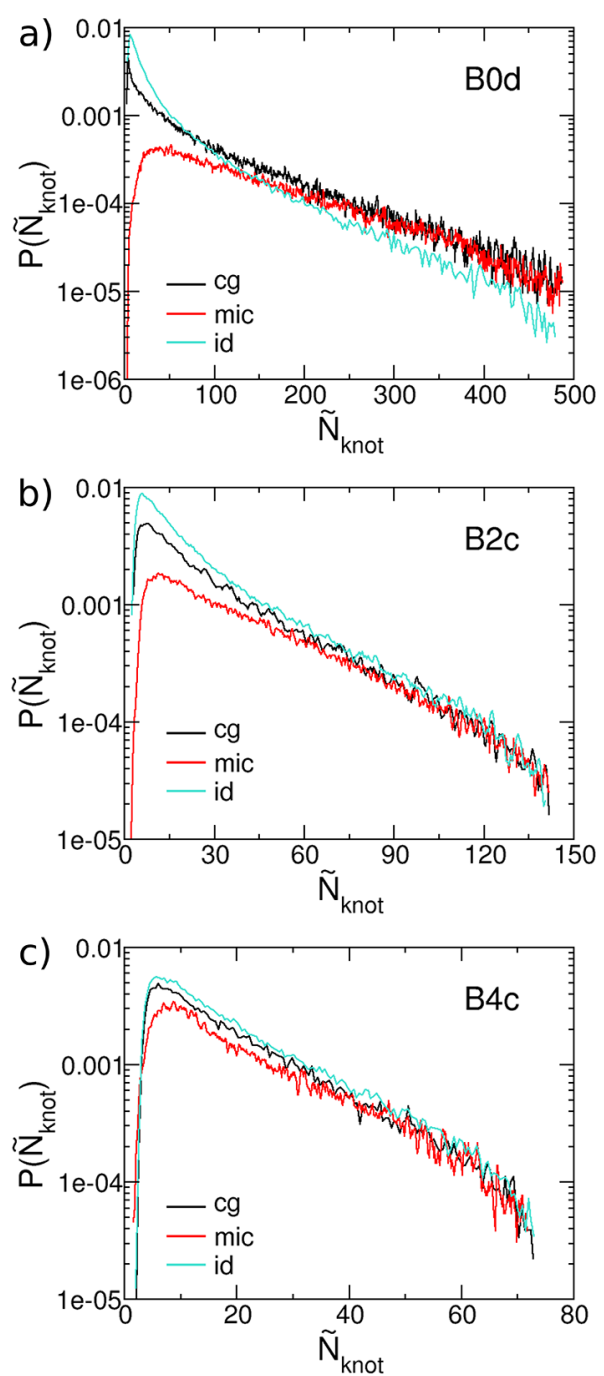

Figure 9. Comparison of distributions $P\left(\tilde{N}_{\mathrm{knot}}\right)$ of the size of trefoil knots in mesoscopic (black) melts, corresponding reference (red) melts, and equivalent ideal Kuhn chains (light blue). The plots address cases corresponding to (a) B0d, (b) B2c, and (c) B4c systems. 
All distributions are normalized such that the integral of $P\left(\tilde{N}_{\text {knot }}\right)$ equals the probability $P_{3_{1}}$ that a randomly selected chain forms a single trefoil knot.

Let us first make some general comments. Figure 9 demonstrates that the "spectra" of trefoil knots in the mesoscopic and reference melts come closer to each other as the polymers become stiffer, in agreement with the free energy model. Moreover, for the stiffest B4 melt the $P\left(\tilde{N}_{\text {knot }}\right)$ in the mesoscopic melts is close to the knot-size distribution in ideal chains. For a more detailed discussion of Figure 9 it is useful to focus on two main features of $P\left(\tilde{N}_{\text {knot }}\right)$ : the size of the most probable knot (peak position) $\tilde{N}_{\mathrm{knot}, \alpha}^{*}$ (where $\alpha=\mathrm{cg}$ or mic indicates the model) and the decay at large values of $\tilde{N}_{\text {knot }}$. These features reflect the behavior of relatively small and large knots, respectively.

We quantify the difference in the size of the most probable knot between mesoscopic and microscopic models via the relative deviation $\delta_{\text {knot }}$ defined as

$$
\delta_{\text {knot }}=\frac{2\left(\tilde{N}_{\mathrm{knot}, \mathrm{mic}}^{*}-\tilde{N}_{\mathrm{knot}, \mathrm{cg}}^{*}\right)}{\tilde{N}_{\mathrm{knot}, \mathrm{mic}}^{*}+\tilde{N}_{\mathrm{knot}, \mathrm{cg}}^{*}}
$$

The results read $\delta_{\text {knot }}=162 \%(\mathrm{~B} 0 \mathrm{~d}), 52 \%(\mathrm{~B} 2 \mathrm{c})$, and $38 \%$ (B4c). They illustrate our generic observation that the agreement between trefoil knot distributions in mesoscopic and reference melts improves significantly in the stiffest B4 melts. In the reference melts $\tilde{N}_{\text {knot }, \alpha}^{*}$ shifts to smaller values as chains become stiffer, which is also consistent with the qualitative predictions from $\beta F_{\text {knot }}$.

We now compare the tails of the trefoil knot size distributions. The free energy model cannot ${ }^{36,58}$ describe large knots because they are no longer tight, and simulations become crucial for understanding their behavior. Figure 9 demonstrates that within the statistical noise of the data the tails of the distributions $P\left(\tilde{N}_{\text {knot }}\right)$ in mesoscopic and reference melts match each other well, even for the most flexible B0d system. However, the tail of $P\left(\tilde{N}_{\text {knot }}\right)$ for ideal chains corresponding to the B0d system differs substantially from the distributions in the mesoscopic and reference melts, suggesting a different functional dependence on $\tilde{N}_{\text {knot }}$. Intriguingly, this is not the case for ideal chains that correspond to the $\mathrm{B} 2 \mathrm{c}$ and $\mathrm{B} 4 \mathrm{c}$ melts-the tail of their $P\left(\tilde{N}_{\text {knot }}\right)$ follows closely the distributions in the melts.

Presumably, ideal chains cannot capture the behavior of large trefoil knots in some of the melts considered in our study because of their small $\sqrt{\bar{N}}$. The small $\sqrt{\bar{N}}$ leads to strong deviations from the Flory hypothesis (cf. section 4.2) so that the ideal-chain approximation to polymer conformations, including their knots, is inaccurate. Indeed, Table 1 demonstrates that even for the longest of the B0 melts, the B0d system, we have only $\sqrt{\bar{N}} \simeq 60$. In contrast, the $\sqrt{\bar{N}}$ of the B2 and B4 melts, considered in Figure 9, are either comparable or significantly larger than $10^{2}$.

We monitor the values of probability $P_{3_{1}}$ in melts of chains having the same contour length $L$ but different stiffness. Table 2 lists $P_{3_{1}}$ for a representative set of such microscopic melts B0c, B2c, and B4c as well as for their mesoscopic counterparts. The data points are certainly too few to accurately trace the dependence of $P_{3}$ on stiffness, but they demonstrate that it is nonmonotonous. Clearly, for the melts in Table $2 P_{3_{1}}$ has a maximum at some stiffness $B_{\max }$ where $B_{\max }<4$. The
Table 2. Probability $P_{3_{1}}$ That a Randomly Selected Chain

Forms a Single Trefoil Knot in Three Melts of Microscopically Resolved Chains (mic), B0c, B2c, and B4c, Presented as a Function of Chain Stiffness Parameter $B$ and $P_{3_{1}}$ Calculated in the Mesoscopic Representations (cg) of These Melts ${ }^{a}$

\begin{tabular}{lccc} 
& B0c & B2c & B4c \\
\hline $\mathrm{mic}$ & 0.0276 & 0.0751 & 0.0638 \\
$\mathrm{cg}$ & 0.0807 & 0.1279 & 0.0881
\end{tabular}

${ }^{a}$ All chains have the same contour length $L=511 b_{\text {mic }}$. For all data the statistical error is below $0.6 \%$.

nonmonotonous effect of chain stiffness on $P_{3_{1}}$ in our multichain melts is qualitatively consistent with the findings of previous studies ${ }^{43,44}$ in single self-avoiding ring polymers. However, whereas for single chains or rings this maximum is typically located ${ }^{33,43}$ somewhere between stiffnesses $B=4$ and 10 , in melts it occurs somewhat earlier, presumably because of the screening of excluded volume interactions beyond the Edwards correlation length.

It is instructive to juxtapose the trends in knotting behavior with the deviations observed in the internal distance plots (Figure 5b). For the B0 and B2 melts, we notice that for chemical distances comparable to the size of the most probable knot in the microscopic model the deviations of internal distance plots of the mesoscopic melts from the reference data are well below $3 \%$. Nevertheless, the knotting properties of the two systems on these scales differ substantially. This observation suggests that for melts of flexible chains knot spectra provide additional quantifiers of the accuracy with which a coarse-grained model reproduces the conformational properties of a target system.

Figure 9 is consistent with our conclusion that the stiffer the chains in microscopic melts, the better the agreement between knotting properties in mesoscopic and microscopic models. However, the plots in Figure 9 refer to trefoil knots only. Therefore, it is important to obtain evidence that our conclusion holds for more complex knots.

Therefore, we perform mesoscopic simulations of all reference melts listed in Table 1 , setting $\nu_{\kappa}=3$ and $\eta=1$. For all these melts we calculate the knotting probability $P_{\mathrm{k}}-\mathrm{a}$ quantity that encapsulates contributions from all possible knot types. Figure 10a presents $P_{\mathrm{k}}$ as a function of $N_{\mathrm{K}}$ in mesoscopic simulations and reference B0, B2, and B4 systems. For all chain lengths and stiffness parameters, the knotting probability in the mesoscopic simulations is higher than in the reference systems. However, in agreement with the qualitative predictions from $\beta F_{\text {knot }}$ these deviations decrease as chains become stiffer. Moreover, the $P_{\mathrm{k}}$ of the stiffest chains, the B4 systems, are close to the knotting probabilities of the ideal chain.

In Figure 10b, we group the data from Figure 10a according to the contour length of the chains and plot $P_{\mathrm{k}}$ as a function of chain stiffness. Within the error bar of the data, we observe that $P_{\mathrm{k}}$ has a nonmonotonous dependence on polymer stiffness for all mesoscopic melts and all microscopic melts shorter than $N_{\text {mic }}=1024$. As is the case of $P_{3_{1}}$ (cf. Table 2), this behavior is qualitatively consistent with the trends found in single selfavoiding ring polymers. ${ }^{43,44}$ 

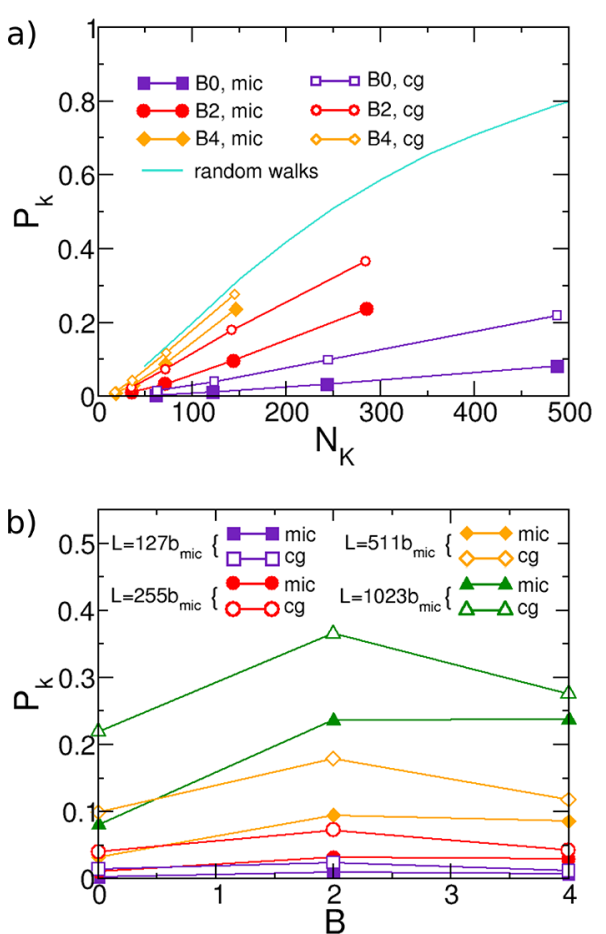

Figure 10. (a) Knotting probability $P_{\mathrm{k}}$ plotted as a function of chain length, expressed in number of Kuhn segments $N_{\mathrm{K}}$ for the reference melts (solid symbols) and their equivalent mesoscopic melts (open symbols). The results for random walks are shown by light blue solid line. (b) The data on $P_{\mathrm{k}}$ from (a) are grouped according to the contour length $L$ of the chains and are plotted as a function of chain stiffness parameter $B$. In all cases the error bar is comparable to the symbol size. The results for the reference melts are reproduced from ref 48 .

\section{CONCLUSIONS}

We focused on the fundamental question whether and under which conditions coarse-grained models with soft interactions can describe knots in polymer melts. We employed a mesoscopic model where polymers are represented by wormlike chains and nonbonded interactions are defined in a way that deliberately creates melts with simplified local liquid structure. The model was parametrized to accurately reproduce mesoscopic structure and conformations of melts that have been modeled earlier ${ }^{48}$ with a microscopic model. The latter captures $^{76}$ generic features of polymer conformations and structure at all length scales and provides, therefore, reliable reference data on knots.

We compared characteristic knotting properties in mesoscopic and reference melts. The simulations realized different scenarios for chain stiffness while keeping the size of monomer-monomer excluded volume fixed. For the simplest possible knot, the trefoil knot, we considered the distributions of knot size (length of chain contour involved in the knot). With regard to general knotting properties, we compared the probability that a chain forms a knotted state.

In a recent study, ${ }^{48}$ the microscopic model was compared with ideal chains. It was shown that the knotting probability and knot spectrum are very different-the difference being the larger the more flexible the chains. Considering that it is impossible for monomers to overlap in the microscopic model, that study ${ }^{48}$ speculated that this difference in knotting behavior might be related to corrections to chain ideality because of incomplete excluded volume screening on short and intermediate scales. Our work refines this picture.

Our central conclusion is that the behavior of knots is not determined by the size of excluded volume alone but by how it compares with the length scale of chain rigidity, e.g., Kuhn segment. For systems where these two length scales separate and the Kuhn segment is significantly larger than the size of excluded volume, the influence of local liquid structure on knotting behavior is marginal. Therefore, in such systems, mesoscopic models and ideal chains can accurately describe knots even though the liquid structure on small scales is simplified or non-existent (ideal chains). Our conclusion is consistent with a free energy model of trefoil knots available in the literature. $^{36,58}$

Interestingly, for flexible chains we observed deviations of knotting properties between mesoscopic and microscopic models even though their other conformational descriptors matched very well. Specifically, the deviations of internal distance plots at chemical distances larger than a few Kuhn segments were less than $2 \%$. This observation is important methodologically because it suggests that knots provide an additional sensitive quantifier of chain conformations.

In summary, our study conveys an encouraging message. Mesoscopic models with soft interactions can be indeed used to study polymer knots in melts where the characteristic length scale of chain stiffness is substantially larger than the size of the monomer-monomer excluded volume. In this way, it is possible to benefit from the computational efficiency of mesoscopic models and study open questions related to fundamental physics of knots in polymers.

\section{ASSOCIATED CONTENT}

\section{Supporting Information}

The Supporting Information is available free of charge at https://pubs.acs.org/doi/10.1021/acs.macromol.0c02079.

Influence of excluded volume on size of the most probable knot; partial radial distribution functions for different $\eta$ (PDF)

\section{AUTHOR INFORMATION}

\section{Corresponding Author}

Kostas Ch. Daoulas - Max Planck Institute for Polymer

Research, 55128 Mainz, Germany; 이이.org/0000-00019278-6036; Email: daoulas@mpip-mainz.mpg.de

\section{Authors}

Jianrui Zhang - Max Planck Institute for Polymer Research, 55128 Mainz, Germany; @ orcid.org/0000-0003-06660811

Hendrik Meyer - Institut Charles Sadron, CNRS UPR 22, Université de Strasbourg, 67034 Strasbourg, France

Peter Virnau - Institut für Physik, Johannes GutenbergUniversität Mainz, 55128 Mainz, Germany; Graduate School of Excellence Materials Science in Mainz, 55128 Mainz, Germany; (1) orcid.org/0000-0003-2340-3884

Complete contact information is available at: https://pubs.acs.org/10.1021/acs.macromol.0c02079

\section{Notes}

The authors declare no competing financial interest. 


\section{ACKNOWLEDGMENTS}

We appreciate helpful discussions with Kurt Kremer and Marcus Müller. We are grateful to Burkhard Dünweg for critical reading of our manuscript and useful comments. We are grateful to the Deutsche Forschungsgemeinschaft (DFG, German Research Foundation) for funding this research: Project number 233630050-TRR 146. H.M. acknowledges a generous grant of CPU time of the High Performance Computing Center of the University of Strasbourg (funded by Equipex Equip@Meso and CPER Alsacalcul/Big Data). P.V. and K.D. also acknowledge the contribution of the COST Action CA17139.

\section{REFERENCES}

(1) Likos, C. N. Effective Interactions in Soft Condensed Matter Physics. Phys. Rep. 2001, 348, 267-439.

(2) Klapp, S. H. L.; Diestler, D. J.; Schoen, M. Why are Effective Potentials "Soft"? J. Phys.: Condens. Matter 2004, 16, 7331-7352.

(3) Müller, M. Studying Amphiphilic Self-assembly with Soft Coarse-grained Models. J. Stat. Phys. 2011, 145, 967-1016.

(4) Noid, W. G. Perspective: Coarse-grained Models for Biomolecular System. J. Chem. Phys. 2013, 139, 090901.

(5) Peter, C.; Kremer, K. Multiscale Simulation of Systems - from the Atomistic to the Coarse-grained Level and Back. Soft Matter 2009, 5, 4357-4366.

(6) Praprotnik, M.; Site, L. D.; Kremer, K. Multiscale Simulation of Soft Matter: from Scale Bridging to Adaptive Resolution. Annu. Rev. Phys. Chem. 2008, 59, 545-571.

(7) Guenza, M. G. Theoretical Models for Bridging Timescales in Polymer. J. Phys.: Condens. Matter 2008, 20, 033101.

(8) de Pablo, J. J. Coarse-Grained Simulations of Macromolecules: From DNA to Nanocomposites. Annu. Rev. Phys. Chem. 2011, 62, 555-574.

(9) Khalatur, P. G.; Khokhlov, A. R. Nonconventional Scenarios of Polymer Self-assembly. Soft Matter 2013, 9, 10943-10954.

(10) Fredrickson, G. H.; Delaney, K. T. Field-theoretic Simulations: An Emerging Tool for Probing Soft Material Assembly. MRS Bull. 2018, 43, 371-378.

(11) Gartner, T. E.; Jayaraman, A. Modeling and Simulations of Polymers: A Roadmap. Macromolecules 2019, 52, 755-786.

(12) Svaneborg, C.; Karimi-Varzaneh, H. A.; Hojdis, N.; Fleck, F.; Everaers, R. Multiscale Approach to Equilibrating Model Polymer Melts. Phys. Rev. E: Stat. Phys., Plasmas, Fluids, Relat. Interdiscip. Top. 2016, 94, 032502

(13) Carbone, P.; Karimi-Varzaneh, H. A.; Müller-Plathe, F. Finegraining without Coarse-Graining: An Easy and Fast Way to Equilibrate Dense Polymer Melts. Faraday Discuss. 2010, 144, 25-42.

(14) Zhang, G.; Moreira, L. A.; Stühn, T.; Daoulas, K. C.; Kremer, K. Equilibration of High Molecular Weight Polymer Melts: A Hierarchical Strategy. ACS Macro Lett. 2014, 3, 198.

(15) Zhang, G.; Chazirakis, A.; Harmandaris, V. A.; Stühn, T.; Daoulas, K. C.; Kremer, K. Hierarchical Modelling of Polystyrene Melts: from Soft Blobs to Atomistic Resolution. Soft Matter 2019, 15, 289-302.

(16) de Gennes, P. Scaling Concepts in Polymer Physics; Cornell University Press: 1979.

(17) Vologodskii, A.; Lukashin, A.; Frank-Kamenetskii, M. Topological Interaction between Polymer Chains. Sov. Phys.JETP 1974, 40, 932-936.

(18) Frank-Kamenetskii, M.; Lukashin, A.; Vologodskii, A. Statistical Mechanics and Topology of Polymer Chains. Nature 1975, 258, 398402.

(19) Koniaris, K.; Muthukumar, M. Knottedness in Ring Polymers. Phys. Rev. Lett. 1991, 66, 2211-2214.

(20) Janse van Rensburg, E.; Sumners, D.; Wasserman, E.; Whittington, S. Entanglement Complexity of Self-avoiding Walks. J. Phys. A: Math. Gen. 1992, 25, 6557-6566.
(21) Mansfield, M. Knots in Hamilton Cycles. Macromolecules 1994 27, 5924-5926.

(22) Deguchi, T.; Tsurusaki, K. Universality of Random Knotting. Phys. Rev. E: Stat. Phys., Plasmas, Fluids, Relat. Interdiscip. Top. 1997, 55, 6245-6248.

(23) Katritch, V.; Olson, W.; Vologodskii, A.; Dubochet, J.; Stasiak, A. Tightness of Random Knotting. Phys. Rev. E: Stat. Phys., Plasmas, Fluids, Relat. Interdiscip. Top. 2000, 61, 5545-5549.

(24) Grosberg, A. Critical Exponents for Random Knots. Phys. Rev. Lett. 2000, 85, 3858-3861.

(25) Orlandini, E.; Stella, A.; Vanderzande, C. Polymer Theta-Point as a Knot Delocalization Transition. Phys. Rev. E: Stat. Phys., Plasmas, Fluids, Relat. Interdiscip. Top. 2003, 68, 031804.

(26) Marcone, B.; Orlandini, E.; Stella, A.; Zonta, F. What is the Length of a Knot in a Polymer? J. Phys. A: Math. Gen. 2005, 38, L15L21.

(27) Virnau, P.; Kantor, Y.; Kardar, M. Knots in Globule and Coil Phases of a Model Polyethylene. J. Am. Chem. Soc. 2005, 127, 1510215106.

(28) Micheletti, C.; Marenduzzo, D.; Orlandini, E.; Summers, D. Knotting of Random Ring Polymers in Confined Spaces. J. Chem. Phys. 2006, 124, 064903.

(29) Sulkowska, J.; Sulkowski, P.; Onuchic, J. Dodging the Crisis of Folding Proteins with Knots. Proc. Natl. Acad. Sci. U. S. A. 2009, 106, 3119-3124.

(30) Boelinger, D.; Sulkowska, J.; Hsu, H.-P.; Mirny, L.; Kardar, M.; Onuchic, J.; Virnau, P. A Stevedore's Protein Knot. PLoS Comput. Biol. 2010, 6, e1000731.

(31) Micheletti, C.; Marenduzzo, D.; Orlandini, E. Polymers with Spatial or Topological Constraints: Theoretical and Computational Results. Phys. Rep. 2011, 504, 1-73.

(32) Reith, D.; Cifra, P.; Stasiak, A.; Virnau, P. Effective Stiffening of DNA due to Nematic Ordering Causes DNA Molecules Packed in Phage Capsids to Preferentially Form Torus Knots. Nucleic Acids Res. 2012, 40, 5129-5137.

(33) Virnau, P.; Rieger, F. C.; Reith, D. Influence of Chain Stiffness on Knottedness in Single Polymers. Biochem. Soc. Trans. 2013, 41, 528-532.

(34) Trefz, B.; Siebert, J.; Virnau, P. How Molecular Knots Can Pass through Each Other. Proc. Natl. Acad. Sci. U. S. A. 2014, 111, 79487951.

(35) Wüst, T.; Reith, D.; Virnau, P. Sequence Determines Degree of Knottedness in a Coarse-Grained Protein Model. Phys. Rev. Lett. 2015, 114, 028102.

(36) Dai, L.; Renner, C. B.; Doyle, P. S. Metastable Tight Knots in Semiflexible Chains. Macromolecules 2014, 47, 6135-6140.

(37) Dai, L.; Renner, C. B.; Doyle, P. S. Origin of Metastable Knots in Single Flexible Chains. Phys. Rev. Lett. 2015, 114, 037801.

(38) Rieger, F. C.; Virnau, P. A Monte Carlo Study of Knots in Long Double-Stranded DNA Chains. PLoS Comput. Biol. 2016, 12, e1005029.

(39) Najafi, S.; Podgornik, R.; Potestio, R.; Tubiana, L. Role of Bending Energy and Knot Chirality in Knot Distribution and Their Effective Interaction along Stretched Semiflexible Polymers. Polymers 2016, 8, 347.

(40) Marenz, M.; Janke, W. Knots as a Topological Order Parameter for Semiflexible Polymers. Phys. Rev. Lett. 2016, 116, 128301.

(41) Shimamura, M.; Deguchi, T. Characteristic Length of Random Knotting for Cylindrical Self-Avoiding Polygons. Phys. Lett. A 2000, 274, 184-191.

(42) Rybenkov, V.; Cozzarelli, N.; Vologodskii, A. Probability of DNA Knotting and the Effective Diameter of the DNA Double Helix. Proc. Natl. Acad. Sci. U. S. A. 1993, 90, 5307-5311.

(43) Coronel, L.; Orlandini, E.; Micheletti, C. Non-Monotonic Knotting Probability and Knot Length of Semiflexible Rings: the Competing Roles of Entropy and Bending Energy. Soft Matter 2017, 13, 4260-4267.

(44) Uehara, E.; Coronel, L.; Micheletti, C.; Deguchi, T. Bimodality in the Knotting Probability of Semiflexible Rings Suggested by 
Mapping with Self-Avoiding Polygons. React. Funct. Polym. 2019, 134, 141-149.

(45) Foteinopoulou, K.; Karayiannis, N. C.; Laso, M.; Kroeger, M.; Mansfield, M. L. Universal Scaling, Entanglements, and Knots of Model Chain Molecules. Phys. Rev. Lett. 2008, 101, 265702.

(46) Laso, M.; Karayiannis, N. C.; Foteinopoulou, K.; Mansfield, M. L.; Kroeger, M. Random Packing of Model Polymers: Local Structure, Topological Hindrance and Universal Scaling. Soft Matter 2009, 5, $1762-1770$.

(47) Trefz, B.; Virnau, P. Scaling Behavior of Topologically Constrained Polymer Rings in a Melt. J. Phys.: Condens. Matter 2015, 27, 354110.

(48) Meyer, H.; Horwath, E.; Virnau, P. Mapping onto Ideal Chains Overestimates Self-Entanglements in Polymer Melts. ACS Macro Lett. 2018, 7, 757-761.

(49) Flory, P. The Configuration of Real Polymer Chains. J. Chem. Phys. 1949, 17, 303.

(50) Wittmer, J. P.; Beckrich, P.; Meyer, H.; Cavallo, A.; Johner, A.; Baschnagel, J. Intramolecular Long-range Correlations in Polymer Melts: the Segmental Size Distribution and its Moments. Phys. Rev. E 2007, 76, 011803.

(51) Wittmer, J. P; Beckrich, P; Johner, A; Semenov, A. N; Obukhov, S. P; Meyer, H; Baschnagel, J Why Polymer Chains in a Melt are not Random Walks. EPL 2007, 77, 56003.

(52) Glaser, J.; Qin, J.; Medapuram, P.; Morse, D. C. Collective and Single-Chain Correlations in Disordered Melts of Symmetric Diblock Copolymers: Quantitative Comparison of Simulations and Theory. Macromolecules 2014, 47, 851-869.

(53) Laradji, M.; Guo, H.; Zuckermann, M. J. Off-lattice Monte Carlo Simulation of Polymer Brushes in Good Solvents. Phys. Rev. E: Stat. Phys., Plasmas, Fluids, Relat. Interdiscip. Top. 1994, 49, 31993206.

(54) Müller, M.; Smith, G. Phase Separation in Binary Mixtures Containing Polymers: A Quantitative Comparison of Single-Chain-inMean-Field Simulations and Computer Simulations of the Corresponding Multichain Systems. J. Polym. Sci., Part B: Polym. Phys. 2005, 43, 934-958.

(55) Daoulas, K. C.; Müller, M. Single Chain in Mean Field Simulations: Quasi-Instantaneous Field Approximation and Quantitative Comparison with Monte Carlo Simulations. J. Chem. Phys. 2006, 125, 184904.

(56) Detcheverry, F.; Kang, H.; Daoulas, K. C.; Müller, M.; Nealey, P.; de Pablo, J. Monte Carlo Simulations of a Coarse Grain Model for Block Copolymers and Nanocomposites. Macromolecules 2008, 41, 4989-5001.

(57) Daoulas, K. C.; Rühle, V.; Kremer, K. Simulations of Nematic Homopolymer Melts using Particle-based Models with Interactions Expressed through Collective Variables. J. Phys.: Condens. Matter 2012, 24, 284121.

(58) Grosberg, A. Y.; Rabin, Y. Metastable Tight Knots in a Wormlike Polymer. Phys. Rev. Lett. 2007, 99, 217801.

(59) Virnau, P. Detection and Visualization of Physical Knots in Macromolecules. Phys. Procedia 2010, 6, 117-125.

(60) Virnau, P.; Mirny, L. A.; Kardar, M. Intricate Knots in Proteins: Function and Evolution. PLoS Comput. Biol. 2006, 2, e122.

(61) Pieranski, P.; Przybyl, S.; Stasiak, A. Tight Open Knots. Eur. Phys. J. E: Soft Matter Biol. Phys. 2001, 6, 123-128.

(62) Dai, L.; Doyle, P. S. Effects of Intrachain Interactions on the Knot Size of a Polymer. Macromolecules 2016, 49, 7581-7587.

(63) Auhl, R.; Everaers, R.; Grest, G. S.; Kremer, K.; Plimpton, S. J. Equilibration of Long Chain Polymer Melts in Computer Simulations. J. Chem. Phys. 2003, 119, 12718-12728.

(64) Zhang, J.; Kremer, K.; Michels, J. J.; Daoulas, K. C. Exploring Disordered Morphologies of Blends and Block Copolymers for LightEmitting Diodes with Mesoscopic Simulations. Macromolecules 2020, 53, 523-538.

(65) Likos, C. N.; Watzlawek, M.; Löwen, H. Freezing and Clustering Transitions for Penetrable Spheres. Phys. Rev. E: Stat. Phys., Plasmas, Fluids, Relat. Interdiscip. Top. 1998, 58, 3135-3144.
(66) Rubinstein, M.; Colby, R. H. Polymer Physics; Oxford University Press: 2003.

(67) Grosberg, A. Y.; Khokhlov, A. R. Statistical Physics of Macromolecules; American Institute of Physics: New York, 1994.

(68) Livadaru, L.; Netz, R. R.; Kreuzer, H. J. Stretching Response of Discrete Semiflexible Polymers. Macromolecules 2003, 36, 3732-3744.

(69) Greco, C.; Jiang, Y.; Chen, J. Z. Y.; Kremer, K.; Daoulas, K. C. Maier-Saupe Model of Polymer Nematics: Comparing Free Energies Calculated with Self Consistent Field Theory and Monte Carlo Simulations. J. Chem. Phys. 2016, 145, 184901.

(70) Müller, M.; Binder, K. Computer Simulation of Asymmetric Polymer Mixtures. Macromolecules 1995, 28, 1825-1834.

(71) Wall, F. T.; Mandell, F. Macromolecular Dimensions Obtained by an Efficient Monte Carlo Method without Sample Attrition. J. Chem. Phys. 1975, 63, 4592-4595.

(72) Kumar, S.; Vacatello, M.; Yoon, D. Off-Lattice Monte Carlo Simulations of Polymer Melts Confined between two Plates. J. Chem. Phys. 1988, 89, 5206-5215.

(73) Mavrantzas, V.; Theodorou, D. Atomistic Simulation of Polymer Melt Elasticity: Calculation of the Free Energy of an Oriented Polymer Melt. Macromolecules 1998, 31, 6310-6332.

(74) Moreira, L.; Zhang, G.; Müller, F.; Stühn, T.; Kremer, K. Direct Equilibration and Characterization of Polymer Melts for Computer Simulations. Macromol. Theory Simul. 2015, 24, 419.

(75) Zhang, G.; Stühn, T.; Daoulas, K. C.; Kremer, K. Communication: One Size Fits All: Equilibrating Chemically Different Polymer Liquids through Universal Long-Wavelength Description. J. Chem. Phys. 2015, 142, 221102.

(76) Kremer, K.; Grest, G. Dynamics of Entangled Linear Polymer Melts: a Molecular Dynamics Simulation. J. Chem. Phys. 1990, 92, 5057. 